\title{
Hearing loss in adults with learning disabilities
}

\author{
Many would benefit from a hearing aid
}

Questionnnaire studies have estimated that about one in 10 adults with learning disabilities has impaired hearing, ${ }^{12}$ but full audiological assessment suggests that four times as many people with learning disabilities have hearing loss severe enough to justify using a hearing aid. ${ }^{3-6}$ Assessment is difficult in these patients, and tests must be tailored to each individual. Basic tests require that the person can understand simple instruction and, importantly, be willing to cooperate with the examiner. Whenever possible, tests involving language are useful as they give some idea of how patients manage within their environment. Performance tests, which require only minimal language skills, entail the use of speech stimuli and pure tones from a free field or warble tone audiometer. Most people who reach this stage will then be able to proceed to audiometry.

Ideally, a team skilled in both audiology and learning disabilities should give these tests, ${ }^{67}$ ensuring that most patients will be tested without recourse to electrophysiological tests. These then will be required by only the most profoundly disabled people - an important consideration as the commonly used test of brain stem evoked responses usually requires general anaesthesia in these patients, leading to difficulties with regard to consent.

The same studies that have shown such a high prevalence of hearing loss in people with learning disabilities have also shown that this population goes largely untested. The problem begins with the staff caring for these patients, who do not readily recognise impairment. But local ear, nose, and throat clinics-lacking the time and specialised skills-may fare no better.

With more disabled people being cared for in the community, hearing problems must be recognised and reliable tests of hearing made available. Unrecognised hearing loss affects behaviour and may underlie much of the difficult behaviour that upsets family, staff, and other residents of houses in the community with support staff. The high frequency hearing loss found so commonly in early middle age in people with Down's syndrome may account for the apparent deterioration in previously normal cooperation. Presbycusis may make people with learning disabilities seem difficult or even senile if it is not detected.

Patients whose problems are identified fall into three broad groups. In the first group are those who welcome a trial of a hearing aid after suitable explanation. Benefiting from the experience, they will continue to use the aid, although they may need reminding. In the second group are those whose disabilities preclude using an aid. With skilled help this group is small. Closely monitored programmes set up by psychologists and speech therapists sometimes succeed in the most unlikely patients.

The third group, comprising people who are unaware of their problem or unwilling to admit it or who are anxious about a new experience, presents the greatest challenge, and the outcome depends largely on the availability of well motivated staff, the cooperation of relatives if patients live at home, and the determination to succeed. Introducing a hearing aid gradually, perhaps by desensitising methods, is necessary. In addition, these patients need a long period of help in inserting the aid at the correct volume and constant supervision to ensure the continuing use of the aid. Supervision of simple tasks such as changing batteries, cleaning moulds, and renewing tubing is also essential. When all such help is regularly available the success rate in this group warrants the expenditure of scarce resources. Recent work, however, shows that people-both disabled and non-disabled - who use aids with obvious benefit for a year or more may then lapse if regular informed supervision is withdrawn. ${ }^{68}$ This may happen, for example, when the care of patients is transferred from one centre to another.

Two problems must therefore be tackled. The first is to identify the large pool of people with impaired hearing among those with learning disabilities. Given extra training, speech therapists specialising in treating such people are probably best placed to lead district teams to tackle this problem. Such teams need to decide how they can work most efficiently and cost effectively, and they need the support of local ear, nose, and throat clinics; those experienced in audiological medicine; audiological scientists; and local technicians in hearing aid departments. Unfortunately, current resources are inadequate to treat those whose problems have already been identified.

The second problem is possibly greater: local supervision of the use of hearing aids. ${ }^{9}$ Once again speech therapists are probably best placed to provide it. Therapists have already suggested some schemes, which entail intensive training of local care staff and others who come in contact with these doubly disabled people. Trainers should come from the district teams. As well as the problems of fitting hearing aids there is the problem of unused aids. In people with learning disabilities these represent an unacceptable waste of a treatment that could enhance an already diminished life.

As people with learning difficulties are progressively entering the community this aspect of their care is justifiably 
arousing concern. Programmes already in place require full support to ensure optimal functioning. Other districts may need encouragement to examine their services for this deserving group of patients. ${ }^{10}$

Honorary Consultant,

SYBIL YEATES

Bethlem Royal and Maudsley Hospitals,

c/o Priority Care Unit,

Services for People With Learning Difficulties,

London SE14 5ER

I National Development Team. Development team for the mentally handicapped: second report. London: DHSS, 1978-1979.
2 Kropka BI, Williams C. The epidemiology of hearing impairment in people with a mental handicap. In: Ellis D, ed. Sensory impairment in mentally handicapped people. London: Crowm Helm, 1986:35-60.

3 Cunningham C, McArthur $\mathrm{K}$. Hearing loss and treatment in young Down's syndrome children. Child Care Health Dev 1981;7:357-74.

4 Yeates S. Medical and otological aspects. In: Ellis D, ed. Sensory impairment in mentally handicapped people. London: Croom Helm, 1986:115-48.

5 Evans PIP. Hearing assessment of mentally handicapped people. Heuring Therapy 1988:9.55-9.

6 Yeates S. Hearing in people with mental handicaps: a review of 100 adults. Mental Handicup 1989:17:33-7.

7 Wilson DN, Hare A. Health care screening for people with mental handicap living in the community. BMF 1990;301:1379-81.

8 Terrell (i. A fair hearing for all. Therapy Weekly 1990;17/18:9.

9 Fortnum HM. Haggard M. A population study of the use/non-use of hearing aids. Br 7 dudiol 1985:19:291-2.

10 Pinney S, Ferris-Taylor R. A structured approach to hearing aid use. Speech Therapy in Practice 1990 Jan:4-5.
Bruce Ames is best known for developing the Ames bacterial mutation test, now widely used as an in vitro assay for detecting potential environmental carcinogens. ${ }^{1}$ Ironically Ames is now at the centre of a controversy concerning the causes of cancer, in which he advocates that environmental exposure to manufactured chemicals may be of limited relevance to human cancer, even when such chemicals are mutagenic in an Ames test and carcinogenic in rodent assays. ${ }^{2-17}$

Ames contends that most human genetic damage arises from the oxidation of DNA during normal metabolism. Moreover, he argues that additional mutagenic exposure from the environment results mainly from tobacco smoke, various natural compounds produced by plants to defend themselves, and products that are formed when food is cooked; compared with these sources, the mutagenic burden imposed by environmental exposure to manufactured chemicals is negligible.

Some of the damaging effects of these hazards are counterbalanced by dietary or other antioxidants (which mainly come from fruit and vegetables) and by various mechanisms for DNA repair. Ames argues that these protective mechanisms are less effective when stem cells are put under conditions of chronic proliferative stress and that exposure to agents that accelerate the rate of stem cell division in human tissues can increase the rate at which somatic mutations "escape" repair and become established. As a consequence he suggests that the most important environmental carcinogens may include some whose chief effect is to cause the chronic division of stem cells.

This reasoning is, in itself, neither new nor controversial. It has long been known, for example, that slowly dividing tissues rarely develop tumours. ${ }^{18}$ The primacy given to mitogenesis has, however, caused controversy. It leads Ames to take issue with the philosophy underlying current procedures for rodent carcinogenicity testing. He believes that administering artificially high doses of chemicals to rats and mice will often lead to false positive results for carcinogenicity. This is because, for many chemical exposures at or near the toxic doses used, injured cells will exhibit a chronic mitogenic response not otherwise observed and will be especially susceptible to cancer induction.

At the considerably lower doses which approximate to levels of human exposure, little or no mitogenic response occurs and therefore many chemicals will be largely or wholly non-carcinogenic. In other words, Ames is questioning a fundamental assumption that is usually made in these experiments - namely, that an approximately linear doseresponse extrapolation from high to low doses is justified.

This leads Ames to disagree with those who advocate, for example, massive efforts to remove trace quantities of synthetic pesticide residues from food or drinking water on the basis of positive animal test results. He has particularly highlighted the potential absurdity of banning certain pesticides such as daminozide (Alar) if the effect of doing so is to increase substantially the total ingestion of mutagenic chemicals from the fungal contaminants and endogenous pesticides in untreated or "organic" crops. ${ }^{17} \mathrm{He}$ is also concerned that overzealous attention to these issues may divert scarce financial resources away from major health risks and cause public confusion about the relative importance of different hazards.

Ames has provoked a vigorous response, the main thrusts of which have been to defend the use of linear dose-response extrapolation and to question the importance of endogenous oxidative DNA damage in carcinogenesis. ${ }^{3579111516}$ His critics also argue that animal and in vitro test results are often the only data available for making informed regulatory decisions. If a chemical causes cancer in rats or mice then there will be concern at human exposures; without evidence to the contrary, an assumption of linear extrapolation in cancer risk is said to be the only prudent option.

Reaching definite conclusions about the regulatory implications of Ames's hypotheses would be premature. Some control of exposure to mutagens and animal carcinogens is necessary (especially in occupational environments). Ames has made us aware that such control may have only a limited impact on the overall burden of cancer.

Ames's view is supported by certain observations from cancer epidemiology. Although tissues that are evolved to divide rapidly may be relatively safe, many important risk factors for human cancer strongly affect cell division. ${ }^{18}$ Most notable are the exogenous and endogenous steroid hormones, which have a crucial role in cancers of the breast, ovary, and endometrium and which act by inducing the proliferation of cells. ${ }^{19}$ In addition, infectious agents such as the hepatitis B virus, Opisthorcis viverrini (liver fluke), and Schistosoma haematobium stimulate cell division and are important risk factors for cancer in developing countries. Even tobacco smoke, which is known to contain many mutagens, may act partly as a result of its chronic inflammatory properties. ${ }^{18}$ Ames's central message is about distinguishing substantial human risks from inconsequential ones. By reopening this debate Ames is asking the medical and scientific community 